\title{
Designing and Evaluating Procedural Instructions with the Four Components Model
}

\author{
Michaël Steehouder \\ University of Twente \\ M.F.Steehouder@Utwente.NL
}

\author{
Hans van der Meij \\ University of Twente \\ meij@edte.Utwente.NL
}

\begin{abstract}
Procedures form the heart of user instructions, tutorials, reference guides, job-aids, online help, way-finding instructions, medicine prescriptions, and so on. This paper summarizes the Four Component Model, which is intended to integrate best practices and research outcomes.
\end{abstract}

Keywords: procedural instructions

\section{Introduction}

The Four Components Model of Procedures distinguishes the following main components of procedural instructions: Goals, Prerequisites, Actions and Reactions, and Unwanted States. The model is eminently practical and easy-to-use for people who design, redesign or evaluate procedural instructions.

\section{General Framework}

Procedures are the heart of many types of user support. Yet, they often fail in helping people reach their goal. We focus on one of the causes: flaws in the design of procedures. Our aim is to assist people in recognizing design flaws in procedures and in (re)designing procedures so that they do what they are intended for. The basic knowledge for these actions comes from understanding "what procedures are made of".

The make-up of procedures can be understood from two complementary theoretical perspectives: systems theory and rhetoric. According to systems theory the events that take place when users try to realize a particular task can be described in terms of states and actions. Thus, procedures must inform users about these states and the actions that change these. An overview of the two is presented in Figure 1.
Desired state:

Prerequisite state:

Interim state:

Unwanted state:

Human actions:

System actions:

External actions:
The goal presented to the user

The condition for moving toward the desired state

The intermediate state or sub goal

The to-be-avoided states (e.g., errors, malfunctions)

The actions taken by the user

The responses of the system

The events or actions from outside (e.g., power shortage) that may affect the system

Figure 1: The Four Components Model

By mixing these states and actions in a meaningful way a useful model of procedures can be constructed. We advance a model in which procedures are seen as structured on the basis of four constituent components: Goals, Prerequisites, Actions and Reactions and Unwanted States. In combination with the design guidelines for each component this model forms a strong basis for analysis, design and redesign of procedures.

Rhetoric comes into the picture as a theory that signals that designers must look beyond the logical view imposed by systems theory. If procedures are to function properly as a support for the user, it is critical that their design is sensitive to audience and context. Among others, this means that there is 
a need to establish source credibility ("selling oneself") and product credibility ("selling the domain") to engage and persuade the user.

\section{Goals}

People frequently face complex tasks that cannot be solved easily. When tasks are likely to overwhelm the user the designer should consider task or goal decomposition. Instead of confronting the user with an unwieldy number of separate action steps the designer can create manageable subgoals which may or may not be presented as separate procedures. Goal decomposition is also an important means in helping users automate procedures.

As the prime motivator for consulting procedural instructions, goals are often signaled in the titles of procedures. These should therefore clearly convey the nature of a goal. In addition, when all titles are seen in relation to one another they can convey the big picture of the tasks that are involved in the use of a program, tool or apparatus.

One of the reasons why people benefit from a goal description lies in the fact that users and designers may differ in the terms they use to refer to a particular goal. A goal description can minimize the confusion caused by such vocabulary differences. In addition, it can persuade the user to trying to achieve a particular goal.

There are often several methods to achieve the same goal. First-time users should be instructed to try or use the ones that are pertinent in the right sequence (namely from simple to complex). In addition, they should be taught to appreciate the differences between methods. This can best be done in a 'use-in practice' situation that optimizes the fit between a goal and the method for achieving that goal (e.g., removing a few characters by pressing the Delete-key and removing several paragraphs by deleting these as text blocks).

Many users engage in explorations to acquaint themselves with a new program. This tendency is so strong that designers should follow suit and build such moments of explorations into their designs. An inclusion of sections for 'guided exploration' positioned at the proper place in a procedure (i.e., at the end) can turn these explorations into valuable learning experiences.
These considerations lead to the following guidelines for presenting goals (only a selection is presented here for illustrative purposes).

In goal decomposition:

- It is useful to break down the desired end-state (goal) into two or more intermediate states (sub goals) when the users must execute many actions

- Keep in mind cognitive limitations. Working memory capacity is easily exceeded. Instructions that users need to memorize should require not more than 3 to 5 operations

- Treat subgoals in the same way as goals, by and large

- The connection between related subgoals should be emphasized by their presentation and there may be a need to add information about prerequisite states

The title of a goal should:

- Be task-oriented

- Reflect the task structure

- Be in gerund-form

- Present the most general action leading to the goal state

\section{Prerequisites}

Prerequisites are conditions that must be satisfied to give the user a reasonable chance to successfully achieve a goal. An obvious prerequisite is that all the required materials should be present. Instructions for simple assembly tasks (e.g., installing a scanner) generally do a good job in this respect by including a 'shopping list' that allows the user to check whether a package is complete. Designers can generally assess this type of prerequisite fairly easily through rational analysis.

The designer's task is more complex when it comes down to dealing with what the user already needs to be able to do or know for following instructions. There are four main ways in which user documentation can deal with this prerequisite. Ordered in a sequence of stepping up the user support, these options are: (a) ignoring, (b) mentioning, (c) referring, and (d) explaining. The prerequisites that we focus on are: conditions, user skills and user knowledge. 
For the prerequisite component of instruction, the following guidelines are important (only a selection is presented here for illustrative purposes).

In designing the conditions:

- Instruct people to activate the prerequisite state in the first action step

- Show or describe the prerequisite state before the action and reaction part of a procedure

- Make chapters modular

- Provide downloadable files

- Mention necessities before the instructions to act

In dealing with prerequisite knowledge of facts:

- Decide whether to ignore, mention, refer or explain

- Minimize the use of jargon

- Refer to explanations of facts in an index

- Use a glossary to facilitate random access readers

- Present necessary facts in a just-in-time fashion

\section{Actions and Reactions}

Instructions tell users how they can achieve tasks. Their purpose is to enable or stimulate user actions. A factor that impacts on the design of instructions is their nature. A distinction can be made between direct instructions and indirect ones. Direct instructions such as job-aids and worked examples present users with clear directions for achieving a well-defined goal, telling them exactly what is needed to achieve a concrete goal. They are presented more or less as prescriptions to assist users in completing these goals easily, quickly and successfully.

Indirect instructions such as tips, exercises and onyour-own sections support user explorations. The goal directedness or nature of the users' actions is often not very clear-cut; there may not be welldefined goals that users should try to achieve. Nor are the actions always well-defined. Indirect instructions tend to be relatively scarce in many types of procedural support. Unless stated otherwise, the discussions below therefore concentrate on direct instructions.

Procedures are filled with intricate action-reaction patterns. There are switches back and forth between user input on the one hand and system reac- tions and occasionally other kinds of feedback on the other. User actions and system reactions form a tandem. Representing the interaction between user actions and system reactions should therefore be a key consideration in design. And this is also why the two are presented jointly in the Action and Reaction component. Although heavily intertwined, user actions and system reactions are also distinct entities with their own, unique presentation.

The following guidelines reflect the criteria for effective Action and Reaction information in instructions (only a selection is presented here for illustrative purposes).

In selecting the format for presenting single instructions:

- The format for a set of instructions determines the range of options for presenting a single instruction

- The basic action step is the preferred choice

- The attunement of user actions and system reactions merits special attention

In presenting the basic action step:

- Use the formula 'action * object'. The action part in the formula should be represented by a verb

- Generally begin with the action verb, unless syntactic considerations suggest otherwise

- Make it stand out from other information types in a procedure. Users should be able to perceive the basic action step easily when scanning the page

- The use of a general case or scenario accommodates to different configurations and affords reuse of instructions, but may complicate task completion

- The use of a specific case can make a task understandable and motivate users to follow the instructions

Feedback about system states:

- Is a vital means of conveying the actionreaction pattern that characterizes humancomputer interaction

- Is especially important when the system's response is invisible to the user, or when such a reaction appears on screen only temporarily

- Is preferably given visually rather than descriptively 
- Can support users in switching attention between input device, manual and screen

- Can support users in identifying and locating screen elements

- Can support users in verifying

\section{Warnings}

People are prone to make mistakes, which is why a model of procedures must concern itself with unwanted states. In the Four Components model these unwanted states are split into two variants: warnings and problem solving information. The two variants share their attention to problems: their prevention, origins, and solutions. In addition, their presentation in procedural instructions is based on a similar model of how to convey information about unwanted states.

The two differ in that warnings come before the fact whereas problem solving information follows afterwards. Warnings should prevent certain actions of the user, or they should alert users to the presence of a more or less serious risk.

They inform people of things that may go wrong and that should be prevented from occurring.

How people process warnings can be captured in an easy to remember 'See-Think-Use' model. First, people must perceive a message. They must literally see it. Next, then they must understand what it means. They must think about its meaning. And finally they must act accordingly. They must apply what they have just learned and use their knowledge to guide their actions.

The design guidelines for the Warnings component of procedures are summarized as follows (only a selection is presented here for illustrative purposes).

To support users using a warning:

- Give direct instructions

- Instruct users about what to do or what to avoid

- Consider using the reverse order of risk description in favor of a use-order presentation

- Positioning it right before or in the action steps, especially when the task is unfamiliar and the risk is high

\section{Problem-solving information}

Users frequently make mistakes. They sometimes even spend as much as $25 \%$ to $50 \%$ of their time on task on problem solving. Mistakes should therefore be seen as a regular, common, to-be-expected occurrence in human-computer interaction. Instructions should follow suit and support users in detecting, diagnosing and solving problems. This is not the case, however. Apart from special trouble shooting guides most instructional procedures do not give problem-solving the attention it deserves.

How people handle problems can be captured in an easy to remember 'Detect-Diagnose-Correct' model which is, of course, quite similar to the 'See-Think-Use' model for warnings. Again, we suggest that there are three main stages. Users must first detect the presence of a problem. They must notice that something is wrong. Next, they may want to understand what is wrong and what could have been the cause of the mistake. Finally they may want to or need to correct the error.

The design guidelines for the Problem-SolvingInformation component of procedures are summarized below (only a selection is presented here for illustrative purposes).

To support users in detecting a problem:

- Present problem solving information in a justin-time fashion (e.g., immediately after an error-prone action)

- Present problem solving information at a rate of about once for every three to five user actions

- Present problem solving information especially in situation in which mistakes do not evoke an error message

- Alert them to configurations that should (not) be as they are

\section{References}

[1] D. K. Farkas, "The logical and rhetorical construction of procedural discourse," Technical communication, vol. 46, pp. 42-54, 1999.

[2] H. Van der Meij, P. Blijleven, and L. Jansen, "What makes up a procedure?" in Content \& Complexity. Information design in software development and documentation, M. J. Albers 
and B. Mazur, Eds. Mahwah, NJ: Erlbaum, 2003, pp. 129-186.

[3] H. Van der Meij and M. Gellevij, "The four components of a procedure," IEEE Transactions in professional communication, vol. 47, 2004.

\begin{abstract}
About the Authors
Michaël Steehouder holds the Chair of Technical Communication at the University of Twente, Enschede, the Netherlands. His research interests include the design of procedural instruction, helpdesk, and rhetorical aspects of technical communication. He published over 150 articles in Dutch and international journals, and text books on professional communication skills, forms design and software manuals. Michaël is chair of STIC, the Dutch society for technical communication, member of IEEE-PCS' AdCom and associate editor of IEEE Transaction on Professional Communication.
\end{abstract}

Hans van der Meij is senior researcher at the department of Instructional Technology from the University of Twente, Enschede, the Netherlands. His research interests include instructional design, minimalism, the development of self-study materials and ICT-use. In the field of technical documentation his work covers the whole spectrum of scientific research (e.g., development of theoretical models, experimental testing and applied research). He has (co-)authored more than 100 scientific publications and is a regular speaker at international conferences. He received several awards for his research on technical communication (including the IEEE Professional Communication Society's Best Transactions Article Award in 1997). 